\title{
Electron and positron induced processes. POSMOL 2013*
}

\author{
Paulo Limão-Vieira ${ }^{1, a}$, Radu Campeanu ${ }^{2}$, Masamitsu Hoshino ${ }^{3}$, Oddur Ingólfsson ${ }^{4}$, Nigel Mason ${ }^{5}$, \\ Yasuyuki Nagashima ${ }^{6}$, and Hajime Tanuma ${ }^{7}$ \\ 1 Laboratório de Colisões Atómicas e Moleculares, CEFITEC, Departamento de Física, Faculdade de Ciências e Tecnologia, \\ Universidade Nova de Lisboa, 2829-516 Caparica, Portugal \\ 2 York University, Department of Physics and Astronomy, Toronto, ON M3J 1P3, Canada \\ 3 Department of Physics, Sophia University, Chiyoda-ku, Tokyo 102-8554, Japan \\ 4 Department of Chemistry, Science Institute, University of Iceland, 107 Reykjavik, Iceland \\ 5 Department of Physical Sciences, The Open University, Walton Hall, Milton Keynes, MK7 6AA, UK \\ 6 Department of Physics, Tokyo University of Science, 1-3 Kagurazaka, Shinjuku, Tokyo 162-8601, Japan \\ 7 Department of Physics, Tokyo Metropolitan University, Hachioji, Tokyo 192-0397, Japan
}

Received 29 July 2014

Published online 25 September 2014 - (C) EDP Sciences, Società Italiana di Fisica, Springer-Verlag 2014

\begin{abstract}
POSMOL 2013, the international meeting on electron and positron induced processes comprising the XVII International Workshop on Low-Energy Positron and Positronium Physics and the XVIII International Symposium on Electron-Molecule Collisions and Swarms, was held at Kanazawa Bunka Hall, Kanazawa, Ishikawa, Japan, from 19-21 July 2013. The XVII Workshop encompassed all aspects of positron, positronium and antiproton interactions with electrons, atoms, molecules and solid surfaces, and topics related to these, whereas the XVIII Symposium encompassed all aspects of electron interactions with molecules in both gaseous and condensed phases. Particular topics include studies of electron interactions with biomolecules, electron induced surface chemistry and the study of plasma processes. Recent research on the study of electron swarms was also highlighted.
\end{abstract}

\section{Introduction}

The POSMOL 2013 meeting attracted to Kanazawa 132 participants (93 regular, 27 students, 12 accompanying), from twenty two countries with over a quarter of those participants postgraduate students. POSMOL accommodated five plenary sessions covering experiments with dense positrons and positronium, single-photon multipleionization of atoms and molecules by multi-electron coincidence spectroscopy, historical progress in the functional study of the effect of metastables on low-temperature plasmas over a wide pressure-range, positron astrophysics and recent advances in low-energy positron interactions with atoms and molecules. The conference hosted sixteen thematic sessions, consisting of forty four progress reports on the most recent experimental and theoretical achievements on low-energy positron and positronium physics and electron-molecule collisions and swarms. The conference also accommodated a poster session, allowing entry to an extra twenty two presentations guaranteeing

\footnotetext{
* Contribution to the Topical Issue "Electron and Positron Induced Processes", edited by Michael Brunger, Radu Campeanu, Masamitsu Hoshino, Oddur Ingólfsson, Paulo Limão-Vieira, Nigel Mason, Yasuyuki Nagashima and Hajime Tanuma.

a e-mail: plimaovieira@fct.unl.pt
}

therefore an opportunity for more fruitful discussions. During the conference both international advisory committee meetings for "EMS" and "POSITRON" were held.

\section{New scientific insights}

This Topical Issue, devoted to recent developments on experimental and theoretical aspects in low-energy positron and positronium physics and electron-molecule collisions and swarms, collects 37 contributions. A study of aliphatic amino acids using simulated vibrational circular dichroism and Raman optical activity spectra has been reported by Ganesan et al. [1]. Carelli et al. [2] presented and discussed the results obtained from ab initio quantum scattering calculations of the response from neutral aromatic species, like $\mathrm{C}_{6} \mathrm{H}_{5}$ (phenyl) and $\mathrm{C}_{6} \mathrm{H}_{4}$ (benzyne, ortho isomer), to low-energy electron collisions. Elastic integral (ICS), momentum transfer (MTCS) and differential cross sections (DCS) for low-energy positron and electron scattering by methylamine were presented by Silva et al. [3] employing a Schwinger multichannel method (SMC). The gravitational behaviour of antihydrogen at rest (GBAR) experiment, which aims at measuring the gravitational behaviour of antimatter as a crucial test of the Weak Equivalence Principle, has been presented by Sacquin [4] on behalf of the 
GBAR collaboration. Pastega et al. [5] have reported integral, differential and momentum transfer cross sections for elastic scattering of low-energy electrons by boron trifluoride molecules $\left(\mathrm{BF}_{3}\right)$ that is a molecule of relevance to both the low-temperature plasma physics community and as a possible detector of neutrons from nuclear processes. Mariazzi et al. [6] showed that AEgIS (Antimatter Experiment: Gravity, Interferometry, Spectroscopy) is an experiment that aims to perform the first direct measurement of the gravitational acceleration constant $g$ of antihydrogen in the Earth's field. To achieve that a cold antihydrogen beam will be produced by a charge exchange reaction between cold antiprotons and positronium excited in Rydberg states. Electron (positron) impact excitation ( $2 p$ state) of lithium at intermediate energies has been investigated by Kamali et al. [7], whereas a review on electron swarm experiments in dense rare gases was presented by Borghesani [8]. Calculations on the elastic ICS, DCS and MTCS for low-energy electron scattering by $n$-pentanol alcohol, in the gas phase, was presented through a Schwinger multichannel method implemented with pseudopotentials [9]. Mukoyama et al. [10] studied $\mathrm{K}$-shell ionization by positrons in the binary-encounter approximation. Yamada et al. [11] reported calculations on energy thresholds for dissociation channels of positronic alkali-metal hydrides, $\left[\mathrm{XH} ; \mathrm{e}^{+}\right](\mathrm{X}=\mathrm{Li}, \mathrm{Na}$, and $\mathrm{K})$, to $\mathrm{XH}+\mathrm{e}^{+}$(positron dissociation), $\mathrm{XH}^{+}+\mathrm{Ps}$ (positronium dissociation), and $\mathrm{X}^{+}+\left[\mathrm{H}^{-} ; \mathrm{e}^{+}\right]$(positronic hydride ion dissociation) using quantum Monte Carlo and high-level ab initio molecular orbital methods, and including the quantum zero-point vibrational energy of all of the particles. A theoretical study on elastic electron collisions with two conformers of the amino acid alanine $\left(\mathrm{CH}_{3} \mathrm{CH}\left(\mathrm{NH}_{2}\right) \mathrm{COOH}\right)$, was reported by Fujimoto et al. [12] by employing the UK molecular R-matrix codes. Ionization cross-sections for positron collisions with $\mathrm{N}_{2}$ and collimated positronium production from gases were shown by Cooke et al. [13] and Shipman et al. [14], respectively. Takahashi et al. [15] reported on doubly excited states of molecular hydrogen by scattered electronion coincidence measurements, whilst Morandi et al. [16] described a study of positronium thermalization in porous materials.

Electron transport in $\mathrm{CF}_{3} \mathrm{I}$ and $\mathrm{CF}_{3} \mathrm{I}-\mathrm{N}_{2}$ mixtures, a method to obtain static potentials for electron-molecule scattering, and velocity slice imaging studies on dissociative electron attachment to $\mathrm{CF}_{4}$, were delivered by Kawaguchi et al. [17], Das et al. [18] and Ómarsson et al. [19], respectively. Detailed ab initio results for resonance formation in low energy electron scattering from uracil, obtained with the $R$-matrix method, were presented by Mašín and Gorfinkiel [20], whereas Kita and Tachikawa [21] reported on a theoretical investigation of the binding of a positron to vibrational excited states of the hydrogen cyanide molecule. Experimental studies on electron driven processes in a chlorodifluoroacetic acid methyl ester were presented by Kopyra [22], while dissociative electron attachment to titatinum tetrachloride and titanium tetraisopropoxide were presented by Bjarnason et al. [23]. As far as positronium and positrons are concerned, we note the contributions from Andersen et al. [24], Banković et al. [25], Murtagh [26] and Guessoum [27], on "Positronium formation from porous silica in backscattering and transmission geometries", "Positron transport in $\mathrm{CF}_{4}$ and $\mathrm{N}_{2} / \mathrm{CF}_{4}$ mixtures", "A positron buncher-cooler and Positron astrophysics and areas of relation to low-energy positron physics", respectively. White et al. [28] have reported on transport coefficients of electrons in mixtures of gaseous water and tetrahydrofuran (THF) as calculated using a multi-term solution of the Boltzmann equation. This work suggested that using water as the default substitute, for studies of radiation damage in the human body, could be inaccurate. Meanwhile, Oyamada and Tachikawa [29] presented a multi-component molecular orbital study on positron attachment to alkali-metal hydride molecules by investigating the nature of chemical bonding and dissociation limits of [LiH; e+]. Chin et al. [30] have applied the coupledchannel optical method (CCOM) to study the scattering of electrons on rubidium atoms at $20 \mathrm{eV}$.

Finally, we note a set of contributions on electron impact, positron and positronium formation on: (a) "Electron impact fragmentation of thymine: partial ionization cross sections for positive fragments" [31]; (b) "Gas breakdown and secondary electron yields" [32]; (c) "Monte Carlo analysis of ionization effects on spatiotemporal electron swarm development" [33]; (d) "Optimisation of the thickness of the moderator for positron annihilation process study in Ar gas" [34]; (e) "Lowenergy positron scattering from gas-phase uracil" [35]; (f) "Brightness enhancement of a linac-based intense positron beam for total-reflection high-energy positron diffraction (TRHEPD)" [36]; (g) "Interaction of positronium with helium atoms - the classical treatment of the 5-body collision system" [37].

\section{Summary}

We hope that POSMOL 2013 was a positive forum for sharing and developing scientific and technological expertise of current aspects of positron, positronium and antiproton interactions with electrons, atoms, molecules and solid surfaces, and related topics, as well as single electron and electron swarm interactions with molecules in both gaseous and condensed phases. We are looking forward to POSMOL 2015, the XVIII International Workshop on Low-Energy Positron and Positronium Physics and the XIX International Symposium on Electron-Molecule Collisions and Swarms, to be held at the Universidade Nova de Lisboa, Lisboa, Portugal, from 17-20 July 2015.

\section{References}

1. A. Ganesan, M.J. Brunger, F. Wang, Eur. Phys. J. D 67, $229(2013)$

2. F. Carelli, M. Satta, F.A. Gianturco, Eur. Phys. J. D 67, $230(2013)$ 
3. F.M. Silva, M.H.F. Bettega, S.A. Sanchez, Eur. Phys. J. D 68, 12 (2014)

4. Y. Sacquin, Eur. Phys. J. D 68, 31 (2014)

5. D.F. Pastega, R.F. da Costa, M.A.P. Lima, M.H.F. Bettega, Eur. Phys. J. D 68, 20 (2014)

6. S. Mariazzi, S. Aghion, C. Amsler, A. Ariga, T. Ariga, A.S. Belov, G. Bonomi, P. Bräunig, R.S. Brusa, J. Bremer, L. Cabaret, C. Canali, R. Caravita, F. Castelli, G. Cerchiari, S. Cialdi, D. Comparat, G. Consolati, L. Dassa, J.H. Derking, S.D. Domizio, L.D. Noto, M. Doser, A. Dudarev, A. Ereditato, R. Ferragut, A. Fontana, P. Genova, M. Giammarchi, A. Gligorova, S.N. Gninenko, S.D. Hogan, S. Haider, E. Jordan, L.V. Jørgensen, T. Kaltenbacher, J. Kawada, A. Kellerbauer, M. Kimura, A. Knecht, D. Krasnický, V. Lagomarsino, S. Lehner, C. Malbrunot, V.A. Matveev, F. Merkt, F. Moia, G. Nebbia, P. Nédélec, M.K. Oberthaler, N. Pacifico, V. Petráèek, C. Pistillo, F. Prelz, M. Prevedelli, C. Regenfus, C. Riccardi, O. Røhne, A. Rotondi, H. Sandaker, P. Scampoli, J. Storey, M.A.S. Vasquez, M. Špaèek, G. Testera, R. Vaccarone, F. Villa, E. Widmann, S. Zavatarelli, J. Zmeskal, Eur. Phys. J. D 68, $41(2014)$

7. M.Z.M. Kamali, J.H. Chin, S.Y. Ng, K. Ratnavelu, Eur. Phys. J. D 68, 50 (2014)

8. A.F. Borghesani, Eur. Phys. J. D 68, 62 (2014)

9. E.M. de Oliveira, M.T. do N. Varella, M.H.F. Bettega, M.A.P. Lima, Eur. Phys. J. D 68, 65 (2014)

10. T. Mukoyama, K. Tökési, Y. Nagashima, Eur. Phys. J. D 68, $64(2014)$

11. Y. Yamada, Y. Kita, M. Tachikawa, M.D. Towler, R.J. Needs, Eur. Phys. J. D 68, 63 (2014)

12. M.M. Fujimoto, J. Tennyson, S.E. Michelin, Eur. Phys. J. D 68, 67 (2014)

13. D.A. Cooke, D.J. Murtagh, G. Laricchia, Eur. Phys. J. D 68, 66 (2014)

14. M. Shipman, S.J. Brawley, L. Sarkadi, G. Laricchia, Eur. Phys. J. D 68, 75 (2014)

15. K. Takahashi, Y. Sakata, Y. Hino, Y. Sakai, Eur. Phys. J. D 68, 83 (2014)

16. O. Morandi, P.-A. Hervieux, G. Manfredi, Eur. Phys. J. D 68, $84(2014)$

17. S. Kawaguchi, K. Satoh, H. Itoh, Eur. Phys. J. D 68, 100 (2014)
18. T. Das, A.D. Stauffer, R. Srivastava, Eur. Phys. J. D 68, $102(2014)$

19. F.H. Ómarsson, E. Szymańska, N.J. Mason, E. Krishnakumar, O. Ingólfsson, Eur. Phys. J. D 68, 101 (2014)

20. Z. Mašín, J.D. Gorfinkiel, Eur. Phys. J. D 68, 112 (2014)

21. Y. Kita, M. Tachikawa, Eur. Phys. J. D 68, 116 (2014)

22. J. Kopyra, Eur. Phys. J. D 68, 202 (2014)

23. E.H. Bjarnason, B. Ómarsson, S. Engmann, F.H. Ómarsson, O. Ingólfsson, Eur. Phys. J. D 68, 121 (2014)

24. S.L. Andersen, R.R. Johansen, J.B. Overgaard, J.K. Mortensen, K.K. Andersen, H.D. Thomsen, M.D. Lund, J. Chevallier, H. Knudsen, U.I. Uggerhøj, Eur. Phys. J. D 68, $124(2014)$

25. A. Banković, S. Dujko, S. Marjanović, R.D. White, Z.Lj. Petrović, Eur. Phys. J. D 68, 127 (2014)

26. D. Murtagh, Eur. Phys. J. D 68, 213 (2014)

27. N. Guessoum, Eur. Phys. J. D 68, 137 (2014)

28. R.D. White, M.J. Brunger, N.A. Garland, R.E. Robson, K.F. Ness, G. García, J. de Urquijo, S. Dujko, Z.Lj. Petrović, Eur. Phys. J. D 68, 125 (2014)

29. T. Oyamada, M. Tachikawa, Eur. Phys. J. D 68, 231 (2014)

30. J.H. Chin, K. Ratnavelu, Y. Zhou, Eur. Phys. J. D 68, 141 (2014)

31. P.J.M. van der Burgt, F. Mahon, G. Barrett, M.L. Gradziel, Eur. Phys. J. D 68, 151 (2014)

32. D. Marić, M. Savić, J. Sivoš, N. Škoro, M. RadmilovićRadjenović, G. Malović, Z.Lj. Petrović, Eur. Phys. J. D 68, 155 (2014)

33. S. Dujko, Z.M. Raspopović, R.D. White, T. Makabe, Z.Lj. Petrović, Eur. Phys. J. D 68, 166 (2014)

34. T. Oka, Y. Sano, Y. Kino, T. Sekine, Eur. Phys. J. D 68, $156(2014)$

35. J. Franz, F.A. Gianturco, I. Baccarelli, Eur. Phys. J. D 68, 183 (2014)

36. M. Maekawa, K. Wada, Y. Fukaya, A. Kawasuso, I. Mochizuki, T. Shidara, T. Hyodo, Eur. Phys. J. D 68, 165 (2014)

37. K. Tökési, R.D. DuBois, T. Mukoyama, Eur. Phys. J. D 68, 255 (2014) 